\title{
Optimal Single-Session Flow Routing for Wireless Sensor Networks
}

\author{
Y. Thomas Hou ${ }^{\dagger} \quad$ Yi Shi $^{\dagger} \quad$ Jianping Pan ${ }^{\ddagger} \quad$ Scott F. Midkiff ${ }^{\dagger}$ \\ ${ }^{\dagger}$ Virginia Tech, The Bradley Department of Electrical and Computer Engineering, Blacksburg, VA, USA. \\ $¥$ University of Waterloo, Waterloo, Ontario, Canada.
}

\begin{abstract}
Wireless sensor networks are becoming increasingly important in recent years due to their ability to detect and convey real-time in-situ scenes for many civil and military applications. A major technical challenge for a wireless sensor network lies in the energy constraint at each node, which poses a fundamental limit on the network lifetime. We consider two-tiered wireless sensor networks and address the network lifetime problem for upper-tier aggregation and forwarding nodes (AFNs). Prior efforts have formulated the network lifetime problem into a linear programming (LP) that results in a multi-session flow routing solution. Under multi-session flow routing, each AFN must be equipped with multiple transmitter to reach various destinations at the same time, which poses a scalability problem in practice. This paper shows that, by exploiting dynamic power control at an AFN's transmitter, a multi-session flow routing solution can be converted into an equivalent single-session flow routing solution (requiring only a single transmitter at each AFN). Consequently, it shows that it is possible to design a single-session flow routing solution that has the same optimal network lifetime as a multisession flow routing solution.
\end{abstract}

Index Terms-Network lifetime, energy constraint, flow routing, power control, wireless sensor networks.

\section{INTRODUCTION}

Wireless sensor networks have recently found many new applications that could have significant impact throughout our society. In this paper, we consider two-tiered wireless sensor networks that can be deployed for various sensing applications. These networks consist of a number of sensor clusters and a base-station. Each cluster is deployed around a strategic location and consists of a number of wireless application sensor nodes (ASNs) and one aggregation and forwarding node (AFN). Each ASN is used to capture and transmit data streams to an AFN while the AFN performs in-network processing by aggregating all correlated information within the cluster (which is also known as "fusion"). The AFN then forwards the composite data stream to the base-station via single or multihop transmission.

The most important performance measure for wireless sensor networks is network lifetime. For two-tiered wireless sensor networks, whenever an AFN runs out of energy, the sensing capability for that local area is lost. Therefore, the definition for network lifetime would be the time until any AFN fails. Since the lifetime of each individual AFN heavily depends on

This research is supported in part by ONR under grant number N00014-03$1-0521$. its energy consumption behavior, and the majority of power consumption at an AFN is due to its radio communication, it is essential to devise strategies that can minimize radio-related power consumption. One promising approach to maximizing network lifetime is to control the power level of radio transmitter. Since the power level of a radio transmitter directly affects its coverage, it is important to utilize the relaying capability among the AFNs to forward data streams.

This paper investigates the optimal network flow routing (through power control) among the upper-tier AFNs to maximize the network lifetime. Among most of related efforts in this area (see, e.g., [3]), there has been no consideration on the limit of available transmitters at each AFN. Generally, this approach would require the number of transmitters on each AFN be $O(N)$, where $N$ is the number of AFNs in the network. In this paper, we are interested in exploring flow routing solutions by limiting each AFN to be equipped with only a single pair of transmitter/receiver. Under the proposed approach, each AFN can only transmit to a single destination at any time, and thus it is called single-session flow routing solution. There are several reasons why we are interested in such single-session solutions. The single-session solution requires to fully exploit dynamic power control capability at each AFN, which is a much more challenging and interesting problem than static multi-session flow routing solutions that have been studied in prior efforts. More important, we believe that single-session solution (based on dynamic power control) is the foundation to all related research efforts in optimal flow routing problems for maximizing network lifetime.

The main contribution of this paper is the development of a conversion algorithm that transforms a multi-session flow routing solution to an equivalent single-session flow routing solution. The main idea for this conversion algorithm is to exploit dynamic power control capability at each AFN's transmitter. By "equivalence", we mean that, the network lifetime under either solution is the same and the remaining energy on each AFN is also the same at the end of network lifetime. Consequently, this result shows that a single-session flow routing solution can be as powerful as a multi-session flow routing solution in achieving optimal network lifetime.

The remainder of this paper is organized as follows. In Section II, we present the reference network model for the wireless sensor network and discuss its power dissipation behavior. We also formulate the optimal network flow routing problem for 
maximizing network lifetime into an LP problem. Section III presents the conversion algorithm that transforms a multisession flow routing solution to an equivalent single-session flow routing solution. In Section IV, we present an example to illustrate the conversion algorithm. Section V reviews related work and Section VI concludes this paper.

\section{System Modeling And Definitions}

\section{A. Reference Network Model}

We focus on a two-tiered architecture for wireless sensor networks. ${ }^{1}$ Figures 1(a) and (b) show the physical and hierarchical network topology for such a network, respectively. There are three types of nodes in the network: application sensor nodes (ASNs), aggregation and forwarding nodes (AFNs), and basestation (BS). The ASNs constitute the lower-tier of the network. They are deployed in groups (or clusters) at strategic locations for various sensing applications. Each ASN is small and lowcost; they are densely deployed within a small geographical area. The objective of an ASN is very simple: once triggered by an event (e.g., detection of motion or biological/chemical agents), it starts to capture live information (video, audio, or scalar measurement), which it sends directly to the local AFN in one hop. It is worth pointing out that multi-hop routing among the ASNs are not necessary due to the small distance between an ASN and its AFN. By deploying these inexpensive ASNs densely in clusters, and within proximity of a strategic location, it is possible to obtain a comprehensive view of the area by exploring the correlation among the scenes collected at each ASN. Furthermore, the reliability of area surveillance can also be improved through redundancy among the ASNs in the same cluster.

For each cluster of ASNs, there is one AFN, which is different from an ASN in terms of both its physical properties and logical functions. The primary functions of an AFN are: 1) data aggregation (or "fusion") for flows coming from the local cluster of ASNs, and 2) forwarding (or relaying) the aggregated data streams to the next hop AFN toward the base-station. For data fusion, an AFN analyzes the content of each data stream received from ASNs and composes a complete view by exploiting the correlation among each individual scene at the ASNs. In addition to receiving data streams from ASNs within the local cluster and performing fusion function, AFNs have an important networking function for the upper-tier architecture: it serves as the relaying node for other AFNs to forward data streams toward the base-station. Although an AFN is expected to be provisioned with much more energy than an ASN, it also consumes energy at a substantially higher rate (due to wireless communication over large distances). Consequently, an AFN has a limited lifetime. Upon the depletion of energy at an AFN, we expect that the coverage for the particular area under surveillance is lost.

The last component within the two-tiered architecture is the base-station, which is basically the sink node for data streams

\footnotetext{
${ }^{1}$ The two tiered architecture is motivated by recent advances in distributed source coding (DSC) for sensor networks [4], [11].
}

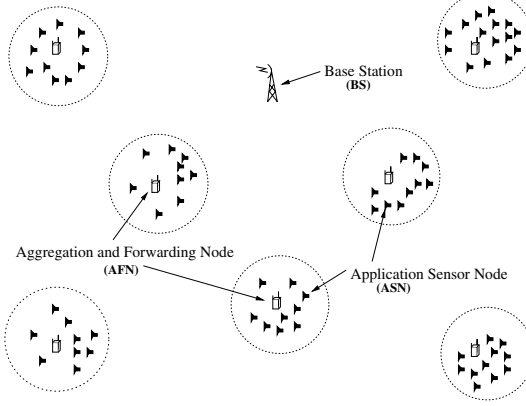

(a) Physical topology.

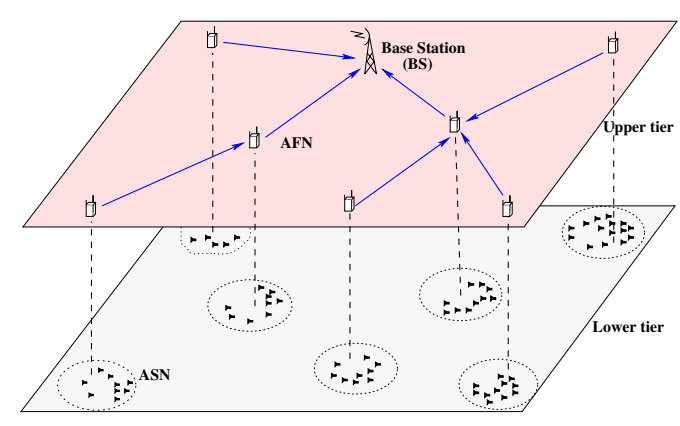

(b) A hierarchical view.

Fig. 1. Reference architecture for a two-tiered wireless sensor network.

generated at all AFNs. A base-station may be assumed to always have a sufficient battery provisioning, or its battery may be re-provisioned during its course of operation. Therefore, its power consumption is not a concern in our investigation.

In summary, the lower-tier ASNs are used for data acquisition. The upper-tier AFNs are used for data fusion and forwarding the aggregated information toward the base-station.

Although the AFNs and base-station locations are immobile, there is a great degree of flexibility in how the network routing topology can be formed to forward information from an AFN toward the base-station. Power control of the transmitter at an AFN can determine radio signal's coverage, which in turn affects the network routing topology [6], [10], [13], [16]. In this paper, we will fully explore dynamic power control capability to develop single-session flow routing solution to maximize network lifetime.

\section{B. Power Consumption Model}

A detailed power dissipation model for each component in a wireless sensor node can be found in [7]. For an AFN, the radiorelated power consumption (i.e., in transmitter and receiver) is the dominant factor [1]. The power dissipation at a transmitter can be modeled as:

$$
p_{t}\left(s_{i}, s_{k}\right)=c_{s_{i}, s_{k}} r_{o},
$$

where $p_{t}\left(s_{i}, s_{k}\right)$ is the power dissipated at AFN $s_{i}$ when it is transmitting to AFN $s_{k}, r_{o}$ is the rate of the data stream sent 
by the transmitter, $c_{s_{i}, s_{k}}$ is the power consumption cost of link $\left(s_{i}, s_{k}\right)$ and

$$
c_{s_{i}, s_{k}}=\alpha_{t 1}+\alpha_{t 2} d_{s_{i}, s_{k}}^{n}
$$

where $\alpha_{t 1}$ is a distance-independent term, $\alpha_{t 2}$ is a distancedependent term, $d_{s_{i}, s_{k}}$ is the distance between the two AFNs, $n$ is the path loss index and $2 \leq n \leq 4$ [12]. Typical values for these parameters are $\alpha_{t 1}=45 \mathrm{~nJ} / \mathrm{b}$ and $\alpha_{t 2}=0.001 \mathrm{pJ} / \mathrm{b} / \mathrm{m}^{4}$ ( $n=4$ ) [2]. Here, we use $n=4$ in all numerical results.

The power dissipation at a receiver can be modeled as [12]:

$$
p_{r}=\alpha_{r} r_{i},
$$

where $r_{i}$ (in $\mathrm{b} / \mathrm{s}$ ) is the incoming rate of received data stream. Typical value of $\alpha_{r}$ is $135 \mathrm{~nJ} / \mathrm{b}$ [2].

\section{Optimal Multi-session Routing via Linear Programming}

In [3], Chang and Tassiulas show that an optimal multisession routing solution can be obtained via a linear programming formulation. We briefly review the technique here.

Let $f_{i k}$ be the rate sent along link $\left(s_{i}, s_{k}\right)$ and $f_{i B}$ be the rate sent along link $\left(s_{i}, B\right)$, we have the following flow balance equation and energy consumption constraint for each AFN,

$$
\begin{gathered}
F_{i}+\sum_{m \neq i} f_{m i}=\sum_{k \neq i} f_{i k}+f_{i B}, \\
\sum_{m \neq i} T f_{m i} \alpha_{r}+\sum_{k \neq i} T f_{i k} c_{i k}+T f_{i B} c_{i B} \leq e_{i} .
\end{gathered}
$$

Our objective is to maximize the network lifetime $T$ while both (4) and (5) are satisfied.

Let $F_{i k}=f_{i k} T$ and $F_{i B}=f_{i B} T$, we have:

Maximize $T$

subject to

$$
\begin{array}{cl}
F_{i} T+\sum_{m \neq i} F_{m i}-\sum_{k \neq i} F_{i k}-F_{i B}=0 & (1 \leq i \leq N) \\
\sum_{m \neq i} \alpha_{r} F_{m i}+\sum_{k \neq i} c_{i k} F_{i k}+c_{i B} F_{i B} \leq e_{i} \quad(1 \leq i \leq N)
\end{array}
$$

where (6) follows from the balance equation (4) and (7) follows from the energy constraint in (5).

We now have a standard LP formulation, i.e., Max $c x$, s.t. $A x \leq b$ and $x \geq 0$, which can be solved in polynomial time [8]. However, such LP approach yields a multi-session flow routing solution, which requires each transmitter to send data streams to multiple receivers at the same time. In the worst case, the number of transmitters on each AFN must be $O(N)$, which is neither scalable nor practical.

In the rest of this paper, we pursue single-session flow routing solutions (requiring each $\mathrm{AFN}$ be equipped with a single pair of transmitter/receiver) that can be as effective as an optimal multi-session solution.

\section{Multi-SESSiOn TO Single-SESSION TRANSFORMATION}

This section present an algorithm that converts a multisession flow routing solution into an equivalent single-session flow routing solution. The main constraint in the conversion is to meet both flow balance equations and energy constraints. The ordering in the transformation algorithm follows a "bottom-up" approach, i.e., from the leaf nodes toward base-station. This approach will ensure that, by the time we perform transformation operation for each AFN, all the nodes from which this AFN receives data streams have already been transformed into single-session mode.

Assume that the system is to be used for time $[0, T]$. During this period, each AFN $s_{i}$ generates a stream at a rate of $F_{i}$, has initial battery level $e_{i}$.

Algorithm 1: (Multi-session to Single-session Flow Transformation (MTS)) Given a multi-session flow routing solution $\psi$ for time $[0, T]$, denote $f_{i k}(t)$ and $f_{i B}(t)$ the flow rates from $\mathrm{AFN} s_{i}$ to $\mathrm{AFN} s_{k}$ and to the base-station $B$ at time $t$, respectively. The following iteration transforms the multisession flow routing solution $\psi$ to an equivalent single-session flow routing solution $\hat{\psi}$.

1) Identify a multi-session AFN $s$ such that

- either $s$ is not receiving data stream from any other AFN (i.e., a leaf node), or

- all AFNs from which AFN $s$ receives data stream are already in single-session mode.

If no such multi-session AFN exists, we already have an equivalent single-session flow routing solution, stop.

2) For AFN $s$, denote $R_{s}=r_{1}, r_{2}, \cdots, r_{\left|R_{S}\right|}$ be the set of immediate relaying (receiving) nodes of $s$ (base-station is considered separately if $s$ sends information to $B$ under $\psi)$. Define $\left|R_{s}\right|+1$ intervals, $T_{r_{1}}=\left[0, t_{1}\right), T_{r_{2}}=\left[t_{1}, t_{2}\right)$, $\cdots, T_{r_{\left|R_{s}\right|}}=\left[t_{\left|R_{s}\right|-1}, t_{\left|R_{s}\right|}\right), T_{B}=\left[t_{\left|R_{s}\right|}, T_{s}\right],{ }^{2}$ such that $T_{r_{k}}\left(k=1,2, \cdots,\left|R_{s}\right|\right)$ and $T_{B}$ satisfy:

$$
\begin{aligned}
\int_{T_{r_{k}}}\left(F_{s}+\sum_{m \neq s} \hat{f}_{m s}(t)\right) d t & =\int_{0}^{T} f_{s, r_{k}}(t) d t, \\
\int_{T_{B}}\left(F_{s}+\sum_{m \neq s} \hat{f}_{m s}(t)\right) d t & =\int_{0}^{T} f_{s B}(t) d t .
\end{aligned}
$$

The network flow routing transformation at $s$ is

$$
\begin{gathered}
\hat{f}_{s, r_{k}}(t)= \begin{cases}F_{s}+\sum_{m \neq s} \hat{f}_{m s}(t) & t \in T_{r_{k}}, \\
0 & \text { otherwise. }\end{cases} \\
\hat{f}_{s B}(t)= \begin{cases}F_{s}+\sum_{m \neq s} \hat{f}_{m s}(t) & t \in T_{B}, \\
0 & \text { otherwise. }\end{cases}
\end{gathered}
$$

3) Go to Step 1).

The following theorem shows the correctness of Algorithm 1. The essence in the proof is to show the following two criteria are met: 1) For each AFN, the incoming (including selfgenerated) and outgoing traffic meet the flow balance equation at any time, i.e., the flow rates are feasible, and 2) at time $T$, the energy consumption at each $\mathrm{AFN}$ is the same before and after the transformation.

Theorem 1: (Multi-session to Single-session Flow Transformation) Suppose that we have a multi-session flow routing solution $\psi$ for time $[0, T]$, Algorithm 1 will give an equivalent single-session flow routing solution $\hat{\psi}$.

\footnotetext{
${ }^{2}$ We will prove $T_{s}=T$.
} 
Proof. We first prove that $T_{s}=T, \int_{0}^{T} \hat{f}_{s k}(t) d t=\int_{0}^{T} f_{s k}(t) d t$, and $\int_{0}^{T} \hat{f}_{s B}(t) d t=\int_{0}^{T} f_{s B}(t) d t$.

If $s$ is a leaf node, then

$$
\begin{aligned}
\int_{0}^{T_{s}} F_{s} d t & =\sum_{k=1}^{\left|R_{s}\right|} \int_{T_{r_{k}}} F_{s} d t+\int_{T_{B}} F_{s}(t) d t \\
& =\sum_{k=1}^{\left|R_{s}\right|} \int_{0}^{T} f_{s, r_{k}}(t) d t+\int_{0}^{T} f_{s B}(t) d t \\
& =\int_{0}^{T}\left(\sum_{k=1}^{\left|R_{s}\right|} f_{s, r_{k}}(t)+f_{s B}(t)\right) d t=\int_{0}^{T} F_{s} d t .
\end{aligned}
$$

The second equality holds by the definitions for $T_{r_{k}}$ and $T_{B}$ in (8) and (9). The last equality holds by flow balance equation at AFN $s$. Therefore, we have $T_{s}=T$. Moreover, we have

$$
\int_{0}^{T} \hat{f}_{s, r_{k}}(t) d t=\int_{T_{r_{k}}} \hat{f}_{s, r_{k}}(t) d t=\int_{T_{r_{k}}} F_{s} d t=\int_{0}^{T} f_{s, r_{k}}(t) d t .
$$

The first equality holds since we only need to consider the time (in integration) when $\hat{f}_{s, r_{k}}(t)$ is non-zero. The second equality holds by flow balance. The last equality holds by applying the definition for $T_{r_{k}}$ in (8). Similarly we can prove that $\int_{0}^{T} \hat{f}_{s B}(t) d t=\int_{0}^{T} f_{s B}(t) d t$.

If $s$ is not a leaf node, then all AFNs from which AFN $s$ receives data stream are already in single-session mode. We now show that $T_{s}=T$. This is true because

$$
\begin{aligned}
& \left.\int_{0}^{T_{s}}\left(F_{s}\right)+\sum_{m \neq s} \hat{f}_{m s}(t)\right) d t \\
= & \sum_{k=0}^{\left|R_{s}\right|} \int_{T_{r_{k}}}\left(F_{s}+\sum_{m \neq s} \hat{f}_{m s}(t)\right) d t+\int_{T_{B}}\left(F_{s}+\sum_{m \neq s} \hat{f}_{m s}(t)\right) d t \\
= & \sum_{k=0}^{\left|R_{s}\right|} \int_{0}^{T} f_{s, r_{k}}(t) d t+\int_{0}^{T} f_{s B}(t) d t=\int_{0}^{T}\left(\sum_{k=0}^{\left|R_{s}\right|} f_{s, r_{k}}(t)+f_{s B}(t)\right) d t \\
= & \int_{0}^{T}\left(F_{s}+\sum_{m \neq s} f_{m s}(t)\right) d t=\int_{0}^{T}\left(F_{s}+\sum_{m \neq s} \hat{f}_{m s}(t)\right) d t .
\end{aligned}
$$

The second equality holds by applying the definitions for $T_{r_{k}}$ and $T_{B}$ in (8) and (9), respectively. The fourth equality holds by the flow balance equation. The last equality holds since we have $\int_{0}^{T} \hat{f}_{m s}(t) d t=\int_{0}^{T} f_{m s}(t) d t$ for AFN $m$. Therefore, we have $T_{s}=T$. Moreover, we have

$$
\begin{aligned}
& \int_{0}^{T} \hat{f}_{s, r_{k}}(t) d t=\int_{T_{r_{k}}} \hat{f}_{s, r_{k}}(t) d t \\
& =\int_{T_{r_{k}}}\left(F_{s}+\sum_{m \neq s} \hat{f}_{m s}(t)\right) d t=\int_{0}^{T} f_{s, r_{k}}(t) d t .
\end{aligned}
$$

The first equality holds since we only need to consider the time (in integration) when $\hat{f}_{s, r_{k}}(t)$ is non-zero. The second equality holds by applying the definition for $\hat{f}_{s, r_{k}}(t)$ in (10). The third equality holds by applying the definition for $T_{r_{k}}$ in (8). Similarly we can prove that $\int_{0}^{T} \hat{f}_{s B}(t) d t=\int_{0}^{T} f_{s B}(t) d t$.

With the above results, we are ready to show that the flow routing solution $\hat{\psi}$ is equivalent to $\psi$. By definitions of $\hat{f}_{s, r_{k}}(t)$ and $\hat{f}_{s B}(t)$ in (10) and (11), the flow balance equation holds at

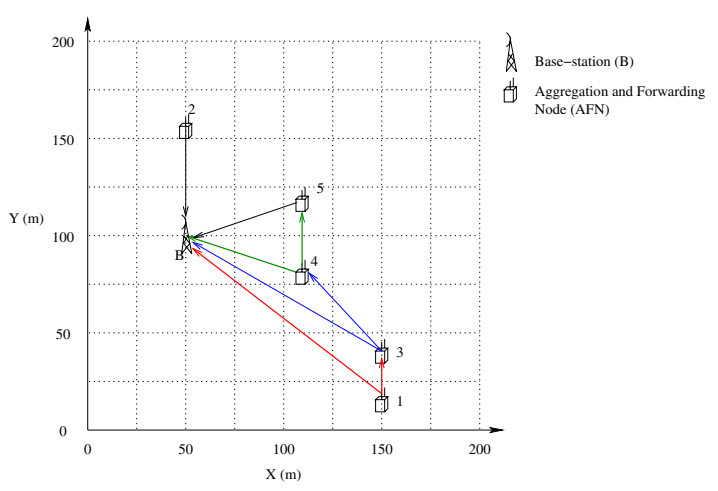

Fig. 2. An example illustrating multi-session to single-session flow routing transformation.

each AFN. All we need to show is that the remaining energy at each AFN at $T$ remain unchanged. For AFN $s$, we have

$$
\begin{aligned}
& \sum_{m \neq s} \alpha_{r} \int_{0}^{T} \hat{f}_{m s}(t) d t+\sum_{k \neq s} c_{s k} \int_{0}^{T} \hat{f}_{s k}(t) d t+c_{s B} \int_{0}^{T} \hat{f}_{s B}(t) d t \\
= & \alpha_{r} \sum_{m \neq s} \int_{0}^{T} f_{m s}(t) d t+\sum_{k \neq s} c_{s k} \int_{0}^{T} f_{s k}(t) d t+c_{s B} \int_{0}^{T} f_{s B}(t) d t \\
\leq & e_{i} .
\end{aligned}
$$

The first equality holds since each corresponding integral term is equal. Thus, at the end of Algorithm 1, we obtain a new network routing solution $\hat{\psi}$ that is equivalent to the network routing solution $\psi$.

The significance of Algorithm 1 is that it can transform any multi-session flow routing solution into a single-session flow routing solution with the same network lifetime. Therefore, an optimal solution developed in the context of single-session flow routing can be as effective as the optimal solution developed under multi-session flow routing.

\section{AN EXAMPLE}

In this section, we use a simple example to illustrate how a multi-session flow routing solution can be transformed into an equivalent single-session flow routing solution.

Example 1: Referring to Fig. 2, suppose that we have 5 AFNs and each AFN has the following coordinates: $s_{1}=$ $(150,20), s_{2}=(50,160), s_{3}=(150,40), s_{4}=(110,80)$, and $s_{5}=(110,120)$ (all in meters $(\mathrm{m})$ ). The base-station $(B)$ is located at $(50,100)$. Suppose that the initial energy and flow rate generated by each AFN are: $e_{1}=1104 \mathrm{~kJ}, F_{1}=360 \mathrm{~kb} / \mathrm{s}$ for $s_{1} ; e_{2}=1040 \mathrm{~kJ}, F_{2}=280 \mathrm{~kb} / \mathrm{s}$ for $s_{2} ; e_{3}=1520 \mathrm{~kJ}$, $F_{3}=200 \mathrm{~kb} / \mathrm{s}$ for $s_{3} ; e_{4}=768 \mathrm{~kJ}, F_{4}=40 \mathrm{~kb} / \mathrm{s}$ for $s_{4}$; and $e_{5}=832 \mathrm{~kJ}, F_{5}=120 \mathrm{~kb} / \mathrm{s}$ for $s_{5}$.

Suppose that we have a multi-session flow routing solution (see Fig. 2) with $f_{i k}$ and $f_{i B}$ shown in Table I. It is easy to verify that the flow balance equation is satisfied at each AFN. For the given initial energy at each AFN, the network lifetime of this flow routing solution is $T=215.04$ days.

We now use Algorithm 1 to transform the above multisession flow routing solution into a single-session flow routing solution. According to Algorithm 1, since $s_{2}$ and $s_{5}$ are already 
TABLE I

INTER-NODAL FLOW RATES IN THE EXAMPLE.

\begin{tabular}{|c|c|c|c|c|c|c|}
\hline & \multicolumn{7}{|c|}{$f_{i k}(\mathrm{~kb} / \mathrm{s})$} & $f_{i B}(\mathrm{~kb} / \mathrm{s})$ \\
\hline$i$ & $k=1$ & 2 & 3 & 4 & 5 & $B$ \\
\hline 1 & 0 & 0 & 199.42 & 0 & 0 & 160.58 \\
\hline 2 & 0 & 0 & 0 & 0 & 0 & 280.00 \\
\hline 3 & 0 & 0 & 0 & 211.55 & 0 & 187.87 \\
\hline 4 & 0 & 0 & 0 & 0 & 191.13 & 60.42 \\
\hline 5 & 0 & 0 & 0 & 0 & 0 & 311.13 \\
\hline
\end{tabular}

in single-session mode, there is no need to perform transformation on these two AFNs (except for the flow rate of $s_{5}$ need to be re-computed). We start with AFN $s_{1}$ and transform it into the following single-session routing: during time $[0,119.12), s_{1}$ sends data stream to $s_{3}$; during time [119.12, 215.04], $s_{1}$ sends data stream to base-station $B$ directly. Following Algorithm 1, we proceed to transform the multi-session flow routing at AFN $s_{3}$ as follows: during time $[0,81.24), s_{3}$ sends data stream to $s_{4}$; during time $[81.24,215.04], s_{3}$ sends data stream to the base-station $B$. Finally, we transform the multi-session routing at AFN $s_{4}$ to single-session and we have: during time $[0,68.50), s_{4}$ sends data stream to $s_{5}$; during time $[68.50,215.04], s_{4}$ sends data stream to the base-station $B$. It is easy to verify that the flow balance equation at each AFN is satisfied throughout time $[0, T]$ and at the time $T$, the energy consumption at each AFN is the same as that under the multisession flow routing solution.

\section{RELATED WORK}

Power control capability has been explored in research at different layers. Here, we briefly review power control at the network (routing) layer, which can be classified into two areas. The first area comprises of strategies to find an optimal transmitter power to control the connectivity properties of the network (see, e.g., [10], [13], [16]). A common theme in these strategies is to adjust each node's transmitter power so that different network connectivity topology can be formed. In [10], Ramanathan and Rosales-Hain's objective is to keep the number of one-hop neighbors be bounded. In [13], [16], the authors aim to design distributed power control algorithms to achieve network connectivity. ${ }^{3}$

The second area could be called power-aware routing. Most schemes use shortest path algorithm with a power-based metric, rather than a hop count based metric (see e.g., [5], [6], [9], [14], [15]). In [14], Singh et al., made some suggestions on metrics for power-aware routing, including energy consumed per-packet, time to network partition, variance in battery life of nodes, cost per packet, and node cost. However, energyaware (e.g., minimum energy path) routing may not ensure good performance in energy-constrained applications. For example, use of the most energy-efficient routes may still result in premature depletion of energy at certain nodes, which is not optimal in terms of network lifetime.

\footnotetext{
${ }^{3}$ The notion of network lifetime in [16] is from connectivity perspective and is different from ours.
}

\section{CONCLUSIONS}

In this paper, we explored single-session flow routing solution to maximize network lifetime for wireless sensor networks. The main contribution of this paper is the development of a conversion algorithm that transforms a multi-session flow routing solution to an equivalent single-session flow routing solution. This result shows that a single-session flow routing solution can be as powerful as a multi-session flow routing solution in achieving maximum network lifetime and thus serves as a capstone for future research in network flow routing for sensor networks.

\section{REFERENCES}

[1] I.F. Akyildiz, W. Su, Y. Sankarasubramaniam, and E. Cayirci, "Wireless sensor networks: A survey," Computer Networks (Elsevier), vol. 38, pp. 393-422, 2002.

[2] M. Bhardwaj and A.P. Chandrakasan, "Bounding the lifetime of sensor networks via optimal role assignments," in Proc. IEEE Infocom, pp. 1587-1596, 2002.

[3] J.-H. Chang and L. Tassiulas, "Energy conserving routing in wireless ad-hoc networks," in Proc. IEEE Infocom, pp. 22-31, Tel Aviv, Israel, March 2000.

[4] J. Chou, D. Petrovis, and K. Ramchandran, "A distributed and adaptive signal processing approach to reducing energy consumption in sensor networks," in Proc. IEEE INFOCOM 2003, April 2003, San Francisco, CA.

[5] S. Doshi, S. Bhandare, and T.X. Brown, "An on-demand minimum energy routing protocol for a wireless ad hoc network," ACM Mibole Computing and Communications Review, vol. 6, no. 3, July 2002.

[6] J. Gomez, A.T. Campbell, M. Naghshineh, and C. Bisdikian, "Conserving transmission power in wireless ad hoc networks," In Proc. IEEE International Conference on Network Protocols, pp. 24-34, Riverside, CA, Nov. 2001.

[7] W. Heinzelman, Application-specific Protocol Architectures for Wireless Networks, Ph.D. thesis, MIT, 2000.

[8] N. Karmarkar, "A new polynomial-time algorithm for linear programming," In Proc. Sixteenth Annual ACM Symp. on Theory of Computing, pp. 302-311, 1984.

[9] Q. Li, J. Aslam, and D. Rus, "Online power-aware routing in wireless Ad-hoc networks," in Proc. ACM MoboCom 2001, pp. 97-107, Rome, Italy.

[10] R. Ramanathan and R. Rosales-Hain, "Topology control of multihop wireless networks using transmit power adjustment," in Proc. IEEE Infocom, pp. 404-413, Tel Aviv, Israel, March 2000.

[11] K. Ramchandran, "Distributed sensor networks: opportunities and challenges in signal processing and communications," presentation at NSF Workshop on Distributed Communications and Signal Processing for Sensor Networks, Dec. 2002, Evanston, IL. Available at http://www.ece.northwestern.edu/ pappas/nsf_work shop/presentations/ramchandran_workshop_DDSP.ppt.

[12] T.S. Rappaport, Wireless Communications: Principles and Practice, Prentice Hall, New Jersey, 1996

[13] V. Rodoplu and T.H. Meng, "Minimum energy mobile wireless networks," IEEE Journal on Selected Areas in Communications, vol. 17, no. 8, pp. 1333-1344, August 1999.

[14] S. Singh, M. Woo, and C.S. Raghavendra, "Power-aware routing in mobile ad hoc networks," in Proc. ACM/IEEE Mobicom, Dallas, TX, Oct. 1998

[15] I. Stojmenovic and X. Lin, "Power-aware localized routing in wireless networks," IEEE Trans. on Parallel and Distributed Systems, vol. 12, no. 11, pp. 1122-1133, Nov. 2001.

[16] R. Wattenhofer, L. Li, P. Bahl, and Y.-M. Wang, "Distributed topology control for power efficient operation in multihop wireless ad hoc networks," in Proc. IEEE Infocom, pp. 1388-1397, 2001. 